\title{
FLUX-LINKAGE CHARACTERISTICS OF SWITCHED RELUCTANCE MOTOR
}

\author{
V. Ramanarayanan \\ Department of Electrical Engineering \\ Indian Institute of Science \\ Bangalore 560012 INDIA
}

\author{
L. Venkatesha \\ Department of Electrical Engineering \\ Indian Institute of Science \\ Bangalore 560012 INDIA
}

\author{
Debiprasad Panda \\ Department of Electrical Engineering \\ Indian Institute of Science \\ Bangalore 560012 INDIA
}

\begin{abstract}
An important requirement in modelling of Switched reluctance motor (SRM) is the knowledge of its flux-linkage characteristics. Idealisation of the flux-linkage characteristics will result in model which cannot predict the performance of the machine with sufficient accuracy. It is necessary therefore to determine flux-linkage characteristics as closely as possible.

In this paper, the method of finding the flux-linkage characteristics is explained. The same is demonstrated on a commercially available $4 \mathrm{KW}, 8 / 6$ pole OULTON SRM. The validity of the test result is checked by computing the Static torque characteristics based on the measured flux-linkage characteristics and comparing it with the experimentally measured Static torque characteristics. The sources of measurement error are discussed.
\end{abstract}

\section{INTRODUCTION}

SRMs are drawing considerable attention for variable speed drive application, owing to its high energy conversion efficiency. It has several advantages over the conventional $\mathrm{AC}$ and $\mathrm{DC}$ drives, namely;

simple and robust construction

brushless operation

low inertia and high torque to weight ratio

simple power converter structure

no shoot through faults between DC buses.

The performance of SRM is influenced by its flux-linkage characteristics, and the control strategy. It is to be stressed that SRM has to be necessarily run from an electronic power converter. A satisfactory control strategy can be developed if accurate knowledge of flux-linkage characteristics of the machine is available.

The flux-linkage in SRM is a function of both rotor position and the current. The family of flux-linkage characteristics (for different rotor positions) will have to be determined as accurately as possible. This paper gives, the method of measurement, sources of experimental error, and precaution required in order to reduce the error.

\section{BASIC PRINCIPLE OF OPERATION}

Figure 1, shows the construction of an $8 / 6$ poles SR motor. It is doubly salient and has no rotor windings. The torque in this motor is due to the tendency of rotor poles to align with the poles of the excited stator phase. The direction of the torque is independent of the direction of the phase current. The phases are cyclically fed by unipolar currents to get unidirectional torque. A shaft position sensor is used to facilitate the turn on and turn off of the phase windings (position sensors can be eliminated with an indirect position sensing scheme).

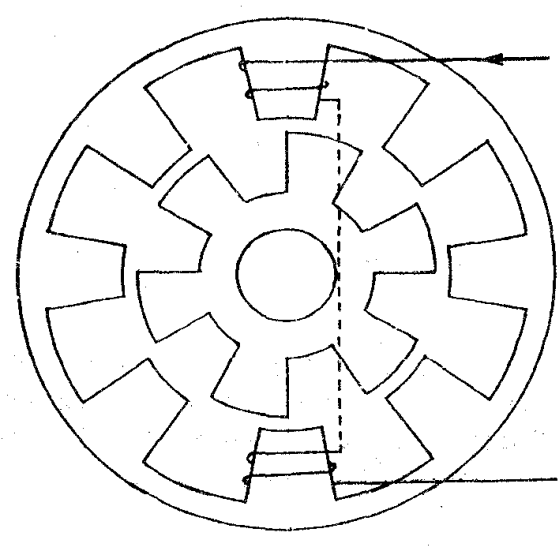

Fig 1 Construction of $8 / 6$ pole SRM

Different modes of control are explained in $[1-2]$.

\section{FLUX-LINKAGE CHARACTERISTICS AND ITS MEASUREMENT}

The flux-linkage characteristics of SRM depends on the rotor position. Due to its doubly salient construction, the machine is prone for saturation. The degree of saturation also depends on the rotor position. The flux-linkage characteristics at different rotor position is explained here in brief. When a pair of rotor poles align with the poles of a stator phase (aligned position), the reluctance for the flux path is at its minimum and the magnetic circuit saturates considerably. The flux-linkage characteristics in aligned position is represented by curve 1 in Fig. 2. When the inter polar axis of the stator coincides with the rotor pole, (unaligned position), the magnetic circuit reluctance will be higher and it is not susceptible for saturation as in aligned position. The flux-linkage characteristics in the unaligned position is as shown in Fig. 2 by curve 3. As, the rotor position is changed from unaligned to aligned position, till the overlap of pole is approached the flux-linkage characteristics do not change appreciably. As the overlap begins, the flux path experiences local saturation in the pole tips and it spreads to the entire magnetic circuit with increase in current The flux-linkage characteristics in the intermediate position is as shown in Fig. 2 by curve 2 . The flux-linkage can be obtained by Finite element methods [3]. The accuracy of the result depends on the skill of the user in choosing the elements. These methods are suitable at design stage and, it is computationally complex. 


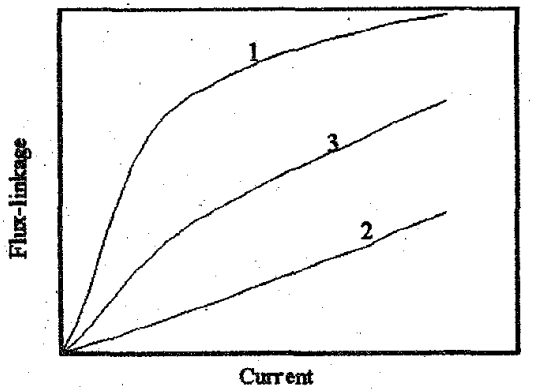

Fig. 2 Fhux-linkage characteristics of SRM at different rotor positions.

The experimental method, which makes use of voltage equation as the basis is discussed in detail. When a voltage pulse is applied to one of the phases of SRM, with all other phases open, its voltage equation is given by,

$$
v=R i+d \Psi / d t
$$

were $v$ is the instantaneous voltage across the phase winding; $R$ is its resistance and $i$ the current. The flux-linkage $\Psi$ is,

$$
\Psi=\int(v-R i) d t
$$

The flux-linkage, can be computed for different values of current with the help of the Eq. (2).

The experimental set-up is as shown in Fig. 3. A machinist's dividing head (indexing head) is used to hold the rotor in position against high torque produced during the experiment. One of the phases of the motor is comnected in series with a rheostat and an electronic switch to a DC source, with all other phases open. A voltage pulse is applied by'turning on the switch. The uration of the voltage applied is made sufficiently large to make the current reach nearly 20 to $30 \%$ more than the estimated peak current encountered during the

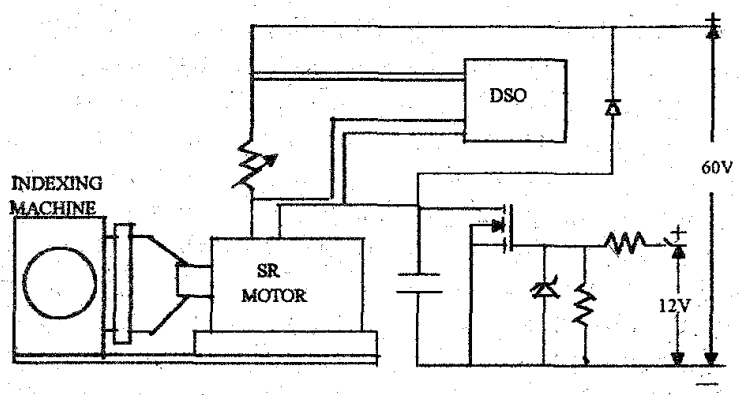

Fig 3 Experimental setup for flux-linkage measurement

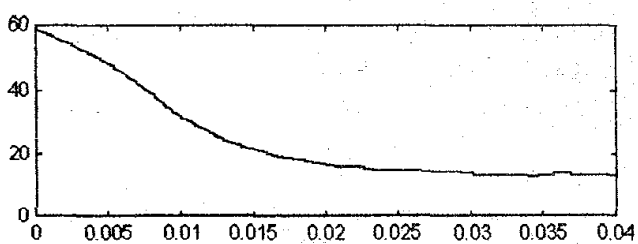

(a)

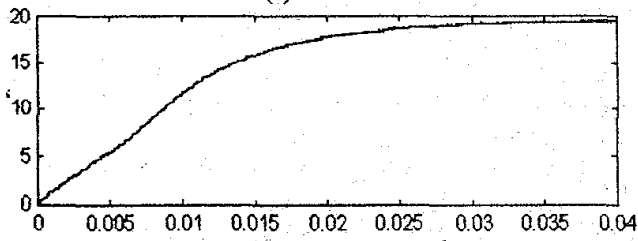

(b)

Fig 4 Voltage and Current waveform

a) versus Time b) Phase Current versus Time

operation. The voltage and the current waveforms are recorded (shown in Fig. 4). The flux-linkage at different current levels are obtained using Eq. (2). The rotor is rotated to a set of equally spaced rotor positions between unaligned to aligned position and procedure is repeated at each rotor position. The flux-linkage characteristics will be symmetric after the aligned position.

Following the above method, flux-linkage characteristics of a $4 \mathrm{KW}, 8 / 6$ pole SRM is obtained. A MOSFET is used as a switching device. An analysing recorder (YOKOGAWA 3556) is used to acquire and store current

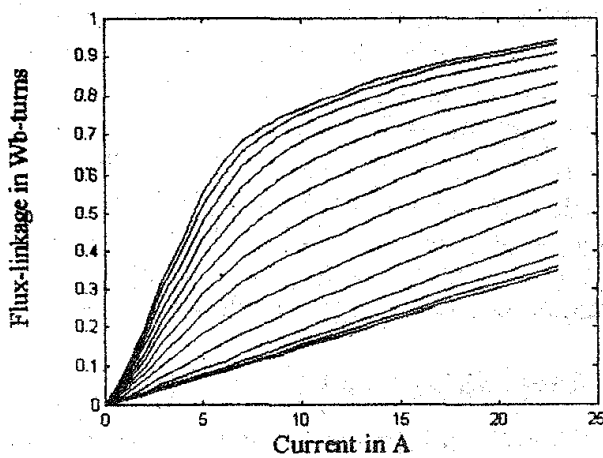

Fig 5 Flux-linkage characteristics of $4 \mathrm{KW}$ SRM

and voltage waveform digitally. The data recorded in Digital Storage Oscilloscope, (DSO) are transferred to the computer and the numerical integration of Eq. (2) is performed using composite Simpson's rule. The results are given in Fig. 5. The validity of these results are checked by evaluating the static torque with the above data and verifying the same by experiment. 


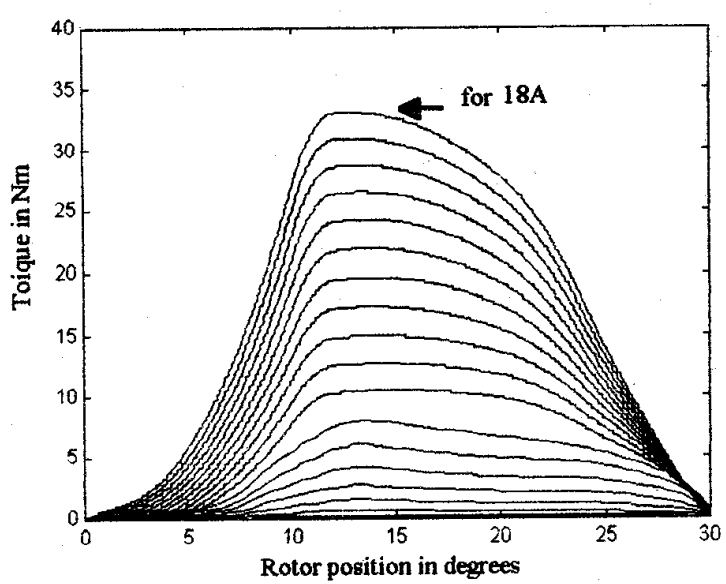

Fig. 6 Computed static torque characteristics

\section{ENERGY CONVERSION PRINCIPLE AND STATIC IORQUE CHARACTERISTICS}

The most general expression for torque produced by one phase at any rotor positions given by, [4]

$$
\mathrm{Te}_{\mathrm{e}}=\left.\frac{\partial \mathrm{W}^{\prime}}{\partial \theta}\right|_{\mathrm{i}=\text { constart }}
$$

where $\mathrm{W}$ is the co-energy. For any given phase current and rotor position, the co-energy is the area under the corresponding magnetisation curve from origin to the point considered This can be expressed as,

$$
\mathrm{W}^{\prime}=\int_{0}^{\mathrm{i}_{1}} \Psi \mathrm{di}
$$

From the Eqs (3), and (4) it is possible to get a set of the torque vs. rotor position characteristics for different current levels. This set of characteristics is known as Static torque characteristics. The Static torque characteristics for the test motor is obtained, first by finding co-energy using spline fit to the flux-linkage characteristics, and then differentiating numerically the co-energy function with respect to rotor position at constant current. A commercially available package is used for this purpose. The results are shown in Fig. 6 .

\section{EXPERTMENTAL MEASUREMENT OF STATIC TOROUE}

The experimental set up is shown in Fig. 7. The set up is almost same as that of Fig. 3 except that a torque transducer is placed in between SRM and the Indexing head. A calibrated Torque transducer (Vibrometer), which has an accuracy of $0.5 \%$, is used to record the torque procuced by the motor. One of the phases of the motor is excited till the desired steady state current flows through the motor, with all other phases open. The torque produced is recorded. The magnitude of the steady state

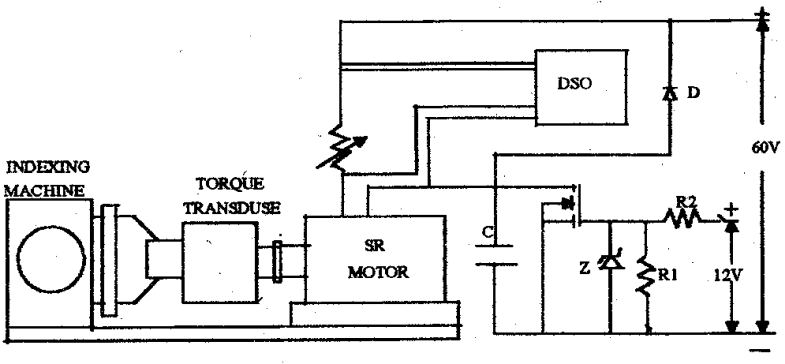

Fig. 7 Experimental setup for the static torque characteristics

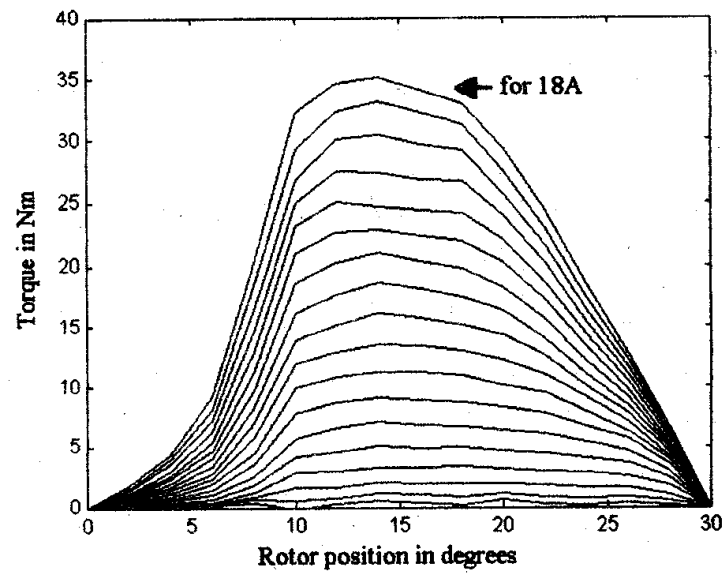

Fig. 8 Experimentally obtained static torque characteristics (for 0 to $18 \mathrm{~A}$ in steps of $1 \mathrm{~A}$ )

current is varied with the help of rheostat, and the corresponding torque are noted. This procedure is repeated for different rotor positions, in the same way as in the flux-linkage characteristics. Static torque characteristics obtained experimentally is shown in Fig. 8.

\section{SOURCES OF ERROR AND ITS INFLUENCE}

\section{Error due to the movement of the rotor}

During the experiment the SRM produces torque higher than the rated nominal torque in certain torque producing regions and it is essential to hold the rotor in position to get reliable results. Change in position during measurement will lead to wrong flux-linkage characteristics corresponding to that angle. If the deviation is considerable it may even lead to intersecting curves for angles closer to aligned position. The influence of rotor motion during the measurement on the instantaneous torque calculation can be explained using the Fig. 9. In figure, curve 1 , and curve 2 are the actual flux-linkage characteristics corresponding to $\theta_{1}$ and $\theta_{2}$ respectively. Let curve 3 be the measured flux-linkage at $\theta_{2}$ with deviation in position during the experiment. The 


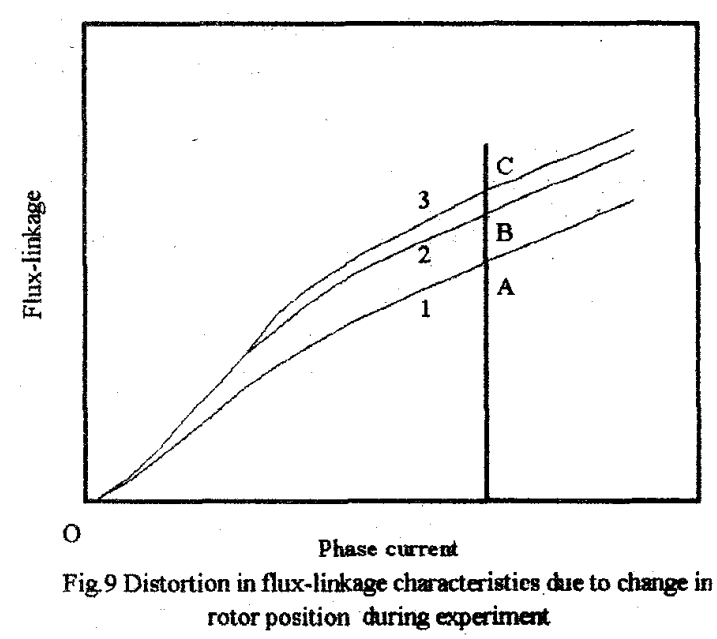

instantaneous torque corresponding to the curve, 2 can be written as,

$\mathrm{T}_{\mathrm{e}}=\frac{\partial \mathrm{W}^{\prime}}{\partial \theta} \mathrm{l}_{\mathrm{i}=\text { constant }}=\frac{\mathrm{OABO}}{\Delta \theta} ;$

where, OABO; change in co-energy for a constant current and for change in angle, $\Delta \theta=\theta_{2}-\theta_{1}$. For the curve 3 the it can be written as,

$\mathrm{T}_{\mathrm{e}}^{\prime}=\frac{\mathrm{OACO}}{\Delta \theta^{\prime}}$

The area $O A C O$ is greater than the area $O A B O$, as the cirve 3 moves up due to the tendency of the rotor to move towards the nearest aligned position. This makes torque to increase for the corresponding position. (if the error in angle in the denominator is not noticed). Also, the current waveform differs from the actual as it is obtained using the same flux-linkage characteristics data. A disc marked in degrees mounted on the shaft and a pointer fixed to the frame of the motor will help in detecting any small movement of the rotor during the experiment. The rotor movement is due to the backlash of the indexing head. Especially in the high torque producing zones the movement is more. This may also increase if the indexing head is moved in the opposite direction around the required point to bring down the angle by a fraction of a degree. If the movement is appreciable during the experiment then an appropriate indexing head with a lesser backlash can be used to hold the rotor in position .

\section{Effect of Numerical integration method}

The flux-linkage can be obtained by integrating ( $v-i R$ ) with respect to time. As DSO stores sampled data after each sampling period, experiments are conducted to determine the influence of the method of integration, and, sampling time on the accuracy of the result. In DSO-

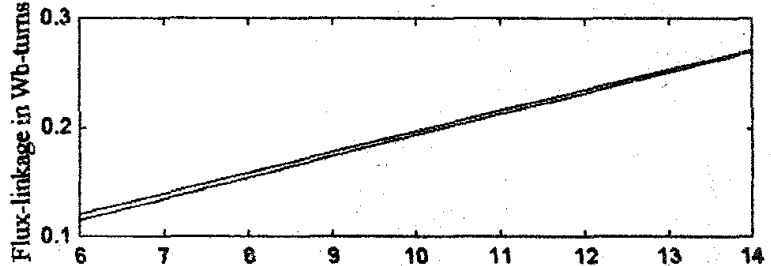

(a)

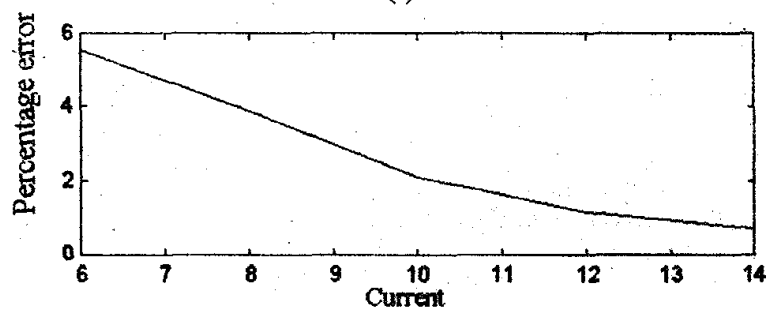

(b)

Fig 10 Effect of edty current on flux-linkage a) Upper curve corresponds to witout eddy current and lower curve corresponds to with eddy current effect

b) Percentage error with eddy currerit effect

YOKOGAWA-3556, the numerical integration is performed as,

Area $=\left[\begin{array}{c}n-1 \\ i=m+1\end{array}(\mathbf{i})+\frac{D(m)+D(n)}{2}\right] \Delta t$

where $\mathbf{m}$ and $\mathrm{n}$ are initial and final points respectively and $\Delta \mathrm{t}$ is the sampling time.

The results obtained using Composite Simpson's rule for integration and that of the DSO did not appreciably differ.

\section{Effect of eddy currents}

When s. e current is rising in the phase winding, all the coupled conducting parts also carry current and changes its flux-linkage. This effect can be minimised by taking measurements with the steady-state current equal to the required current at which the flux-linkage is to be determined, instead computing the flux-linkage at different currents during the transients with a single recording. This is due to the fact that the current in the coupled conducting parts will be zero when the current in the winding attains the steady state. The effect of eddy current on flux-linkage is illustrated in Fig.10. In the lower trace of Fig 10 (a), flux-linkages at different currents are computed using the transient current values of in a single recording with a steady state current of $18 \mathrm{~A}$, whereas upper trace is obtained by conducting several measurements at different steady state currents from 6 to $14 \mathrm{~A}$ in steps of $2 \mathrm{~A}$. Figure $10 \mathrm{~b}$ ) gives the percentage of error at different currents. The error reduces as the current 
increases in the winding. (the maximum error is found to be around $5 \%$,in this case).

Although the results obtained by this method are better, it is tedious and time consuming, as the number of tests to be conducted are higher to get a complete set of flux-linkage characteristics. Presscot bridge method can also be used to eliminate the eddy current effect [5].

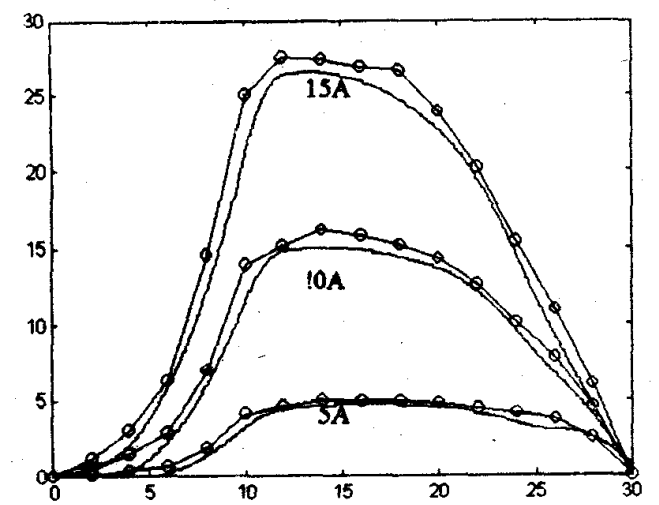

Fig. 11 Comparison between experimental and computed static torque

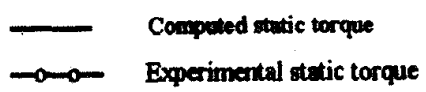

\section{Error due to improper resistance and other factors}

The resistance of the phase winding has to be measured accurately by suitable bridge method. Improper value of resistance can lead to drift in flux-linkage characteristics. The variation of resistance due temperature can introduce error in measurement. This can be minimised by conducting the test, after allowing the machine to cool to the ambient temperature if it is loaded earlier. Measurements are made with sufficient time gap between measurements, so that the temperature effects are minimised.

The effective resistance changes during the experiment cue to the eddy current loss in the coupled conducting parts. This can be taken into account by adapting the resistance arbitrarily in numerical integration, such that when $\mathrm{i}=0, \boldsymbol{\Psi}=0 \quad[4]$.

Error can also occur due to the non linearity in the current transducer. The current probes has to be calibrated and adjusted for any offsets.

\section{COMPARISON AND CONCLUSION}

The Static torque characteristics as measured and as predicted are both shown in Fig. 11. The qualitative match is good. Error in actual numbers is around 10\%, which is within acceptable range for the design purpose. Accuracy can be improved further by taking appropriate measures to rectuce the error influencing factors.

In this paper, a practical way of finding and cross checking of the flux-linkage characteristics of SRM. is described. This is very much useful in simulation of the SR Drive and its control strategy.

\section{REFERENCES}

[1] P.J.Lawrenson, J.M.Stephenson, P.T.Blenkinsop, J.Corda and N.M.Fulton "Variable-speed Switched reluctance motors" IEE PROC. , vol. 127, Pt. B, No. 4, July 1980.

[2] M.R.Harris et al, "Discussions on variable-speed Switched reluctance motor" IEE PROC., vol.128, Pt. B, No. 5, Sept. 1981.

[3] Mehdi Moallem and Chee-Mun Ong " Predicting the steady-state performance of a Switched reluctance machine "IEEE Transactions on I.A. , Vol.27, No.6, Nov/Dec 1991.

[4] T.J.E.Miller "Switched Reluctance Motors and their control " Magna Physics Publishing and Clarendon Oxford 1993

[5] Jones C.V. "The unified theory of electrical machines", Butterworth \& Co., London, 1967. 